\title{
Clinical Study \\ Optimal Positioning for ERCP: Efficacy and Safety of ERCP in Prone versus Left Lateral Decubitus Position
}

\author{
Mashal Batheja, ${ }^{1}$ M. Edwyn Harrison,, ${ }^{2}$ Ananya Das, ${ }^{3}$ Rodney Engel, ${ }^{4}$ and Michael Crowell ${ }^{2}$ \\ ${ }^{1}$ Division of Gastroenterology, Carl T. Hayden VA Medical Center, 650 E. Indian School Road, Phoenix, AZ 85012, USA \\ ${ }^{2}$ Division of Gastroenterology, Mayo Clinic, 13400 East Shea Boulevard, Scottsdale, AZ 85259, USA \\ ${ }^{3}$ Division of Gastroenterology, Arizona Center for Digestive Health, 2680 S. Val Vista, Suite 116, Gilbert, AZ 85295, USA \\ ${ }^{4}$ Division of Gastroenterology, University of New Mexico, 1 University Boulevard of New Mexico, Albuquerque, NM 87131, USA
}

Correspondence should be addressed to Mashal Batheja; mashjatoi@yahoo.com

Received 21 December 2012; Accepted 28 January 2013

Academic Editors: G. Larciprete, A. Mahajna, C. E. Moore, G. von Wichert, and K. Watabe

Copyright (c) 2013 Mashal Batheja et al. This is an open access article distributed under the Creative Commons Attribution License, which permits unrestricted use, distribution, and reproduction in any medium, provided the original work is properly cited.

\begin{abstract}
Background. ERCP is customarily performed with the patient in prone position. For patients intolerant of prone positioning, ERCP in left lateral decubitus (LLD) position offers a potential alternative. Aims. To compare efficacy and safety of ERCP in the LLD position versus prone position. Methods. Consecutive ERCP reports from August 2009 to October 2010 at Mayo Clinic Arizona were reviewed. Inclusion criteria. Age $>18$ years, native papilla, and biliary indication. Primary outcome measure. Bile duct cannulation rate. Secondary outcomes. Times to ampullary localization and bile duct cannulation and complication rate. Results. ERCPs reviewed from 59 patients in two positions: 39 prone and 20 LLD. Cannulation Rate. 100\% prone versus 90\% in LLD $(P=0.11)$. Median (IRQ) times. (1) Ampullary localization: $90 \mathrm{sec}(70-110)$ prone versus $100 \mathrm{sec}(80-118)(P=0.16)$; (2) bile duct cannulation: $140 \mathrm{sec}$ $(45-350)$ prone versus $165 \mathrm{sec}(55-418) \mathrm{LLD}(P=0.54)$. Complications. No periprocedure; postprocedure $4(10 \%)$ prone versus 3 $(15 \%)$ LLD $(P=0.65)$. Conclusion. ERCP performed in LLD position allowed deep bile duct cannulation in $90 \%$ of patients without significantly increased procedural times or rate of complications as compared to prone position.
\end{abstract}

\section{Introduction}

Endoscopic retrograde cholangiopancreatography (ERCP) is conventionally performed in the prone position; this allows for easier passage of scope through the pharynx and low risk of aspiration for the patient and provides an effective and comfortable approach for the endoscopist [1]. However, the prone position is not always optimal or possible, especially for patients with abdominal distension or tenderness due to tense ascites, indwelling percutaneous catheters, morbid obesity, advanced pregnancy, or recent abdominal surgery $[2,3]$. In addition, patients with limited neck mobility who require deep sedation with endotracheal anesthesia may not be able to turn their neck laterally to accommodate the endotracheal tube. Several studies have reported outcomes and safety of ERCP in the supine position as an alternative to standard prone positioning. Ferreira and Baron concluded that an ERCP with the patient in the supine position can be safely and effectively performed if necessitated by clinical circumstances [4], though the supine position can be less comfortable for the endoscopist. This conclusion was different from that reached by the Terruzzi and colleagues who reported that difficult cannulation was markedly increased in the supine position, and cardiopulmonary adverse events were more frequent in this group [5].

The left lateral decubitus (LLD) position provides an alternative position for ERCP in those patients who cannot reliably tolerate prone positioning. While endoscopists may employ the left lateral decubitus position, there has been no formal evaluation of the use of ERCP in this position. Therefore, we undertook a retrospective analysis to compare the efficacy and safety outcomes in patients undergoing ERCP in left lateral decubitus versus prone position.

\section{Methods}

All ERCPs performed at Mayo Clinic Hospital between August 2009 and December 2010 for biliary indications were reviewed. Exclusion criteria included pancreatic indication, prior endoscopic or surgical sphincterotomy, prior partial 
gastrectomy or major surgery of upper GI tract (e.g., total gastrectomy and gastroenteric and bilioenteric anastomoses), and age $<18$ years. Inclusion criteria were patients over 18 years of age undergoing ERCP for biliary indication and not meeting any of the aforementioned exclusion criteria.

ERCP was initiated with the patient in either LLD position or prone position. Patients with significant ascites, indwelling percutaneous catheters, morbid obesity, or limited neck mobility were placed in the LLD position; the remainder of patients did not have medical conditions that would limit their tolerance to routine ERCP positioning and were placed in prone position.

Fluoroscopy was performed using the Siemens Artis zee multipurpose system, model number 07555233, manufactured by Siemens AG in Muenchen, Germany. This unit allows repositioning of the fluoroscopic image by rotating the C-arm tower media-laterally or orbitally and also by a separate tilting mechanism of the table, so that anteriorposterior images can be obtained with patient in LLD position. Specifically, this unit offers the following rotational and tilt capabilities: (1) C-arm media-lateral rotation over a maximum of 210 degrees, from 60 degrees left anterior oblique to 90 degrees right anterior oblique; (2) C-arm orbital rotation in head-to-foot axis over a maximum of 90 degrees, from -45 to +45 degrees; (3) table tilt over a maximum of 180 degrees, from -90 to +90 degrees.

Fluoroscopic technique was performed in consistent fashion throughout the study interval. For those patients situated in prone position, the fluoroscopic unit was positioned initially in anterior-posterior position and then adjusted with media-lateral or orbital rotation as required during the procedure to provide the best images of the biliary ducts of interest. For those patients initially situated in LLD position, the fluoroscopic unit was rotated media-laterally so that images also were obtained in the anterior-posterior plane with respect to the patient. During the course of the procedure, the fluoroscopy unit position was adjusted from this initial position with additional media-lateral or orbital rotation as required to achieve the best images.

Mayo Clinic Institutional Review Board approved the study. In an effort to limit variability and design complexity due to nesting within endoscopist, a single experienced endoscopist (M. E. Harrison) performed all ERCPs. Data were collected prospectively on all procedures and recorded in the endoscopic electronic medical record. Data abstracted included demographics, indication for procedure, type of sedation (moderate sedation with meperidine or fentanyl and midazolam managed by the endoscopist and nurse versus deep sedation managed by the anesthesiologist and/or nurse anesthetist), American Society of Anesthesiology (ASA) risk classification, degree of difficulty of ERCP using Schutz rating criteria [6], patient position, time from esophageal intubation to initial localization of ampulla, time from localization of ampulla to free cannulation of bile duct, cardiac or respiratory complications during the procedure, and complications after the procedure.

Patients were monitored with pulse oximetry and clinical assessment of respiratory status during the procedure and in the recovery area. Significant oxygen desaturation was defined as oxygen desaturation $<85 \%$ of any duration, mask ventilation, and unplanned endotracheal intubation. Hypotension was defined as systolic blood pressure $<90 \mathrm{mmHg}$ requiring use of vasopressor drugs, cardiac arrhythmia (heart rate $>120$ or $<60$ beats per minute) requiring treatment, or premature termination of endoscopy caused by sedation-related events [7]. All complications were noted and documented, including both intraprocedural and postprocedural complications.

The procedural degree of difficulty was determined using the Schutz rating criteria [6] (where $1=$ simple diagnostic ERCP, 2 = simple therapeutic ERCP, 3 = complex diagnostic ERCP, 4 = complex therapeutic ERCP, and 5 = very advanced ERCP).

The primary outcome was biliary cannulation rate in LLD versus prone position. Secondary outcomes were time to initial ampullary localization, time from ampullary localization to deep biliary cannulation, and intra- and postprocedural complication rates.

2.1. Statistical Analysis. Data were entered manually and statistically assessed using the IBM SPSS Statistics Version 19.0, Chicago, IL, USA. Normality of data sets was determined using SPSS Explore and Descriptive functions. Stem-andleaf plots and histograms were used to evaluate variable distributions and assess outliers. Normality plots were used to display normal probability and detrended normal probability plots. Where appropriate, the Kolmogorov-Smirnov statistic was used for testing normality. Frequency distributions were evaluated for all categorical variables (e.g., gender). Student's $t$-test was performed to evaluate differences in demographic variables between groups that were normally distributed. The Mann-Whitney test was used to evaluate differences between groups when the assumptions of normality were not met. Tests for proportionality between groups were made using Chi-square tests for independence. Summary statistics included mean or median and standard deviations (SD) or interquartile range (IRQ) for all variables based on normality of distributions. All significance levels are set to $P<0.05$.

\section{Results}

On review of electronic medical records, 59 patients met inclusion criteria for the study from August 2009 to December 2010. There were 39 patients who underwent ERCP in the prone position and 20 patients had ERCP in the LLD position. The 2 groups did not differ significantly in gender or age. There were 22 females and 17 males in the prone group, with mean age $63 \pm 15$ years. In the LLD group, there were 11 females and 9 males, with mean age $64 \pm 17$ years.

Patients undergoing ERCP in prone and LLD positions were compared with respect to indication for ERCP, ASA classification, and degree of difficulty of ERCP. There were no statistically significant differences between groups in these criteria. The indication for ERCP in both groups was primarily either choledocolithiasis or malignant strictures, and there was no significant difference in indications between groups: $41 \%$ versus $55 \%$ had retained stones and $21 \%$ versus 


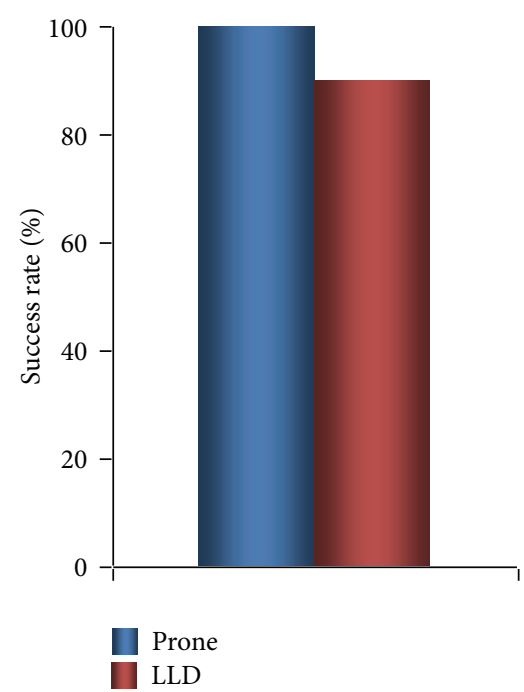

Figure 1: Cannulation success rate in prone and LLD position. The cannulation success rate was $100 \%$ in the prone position and $90 \%$ in $\operatorname{LLD}(P=0.11)$.

$25 \%$ were treated for strictures in prone and LLD groups, respectively. There were no healthy patients in either the prone or LLD group, and a large minority of patients were severely ill or had severe threat to life: in the prone position, $33 \%$ patients were ASA class 3 or greater, while in LLD position, $40 \%$ patients were ASA class 3 or greater (difference not statistically significant). ERCP procedures were almost uniformly therapeutic with the majority of cases designated complex therapeutic cases or advanced therapeutic cases. The Schutz score for difficulty of ERCP was 4 or higher in $72 \%$ of patients in prone position and $65 \%$ of patients in the LLD position; again this difference was not statistically significant. Table 1 details the indications for ERCP, Schutz scores, and ASA classes for patients undergoing ERCP in prone position and LLD position.

The primary outcome of biliary cannulation rate was comparable between patients with ERCP performed in LLD and prone positions. Biliary cannulation was achieved in $18 / 20$ patients $(90 \%)$ in LLD position and $39 / 39$ patients $(100 \%)$ in prone position, and this difference was not statistically significant $(P=0.11)$ (Figure 1$)$. Moreover, although there were two initial failures of biliary cannulation in LLD position, free biliary cannulation was achieved in both of these patients after secondarily converting the patient to prone position and attempting cannulation again.

In the first of the two patients with failed cannulation, LLD position was chosen initially due to ascites, and the ampulla was localized with some difficulty after 2 minutes. However, despite multiple attempts to center the endoscope in both long and short positions, the endoscope could not be stabilized over the ampulla. The patient then was moved into prone position temporarily in spite of the ascites, and in this position the ampulla was readily localized, the endoscope maintained in stable position, and biliary cannulation achieved. For the second patient, LLD position was chosen initially due to significant abdominal pain. The ampulla was

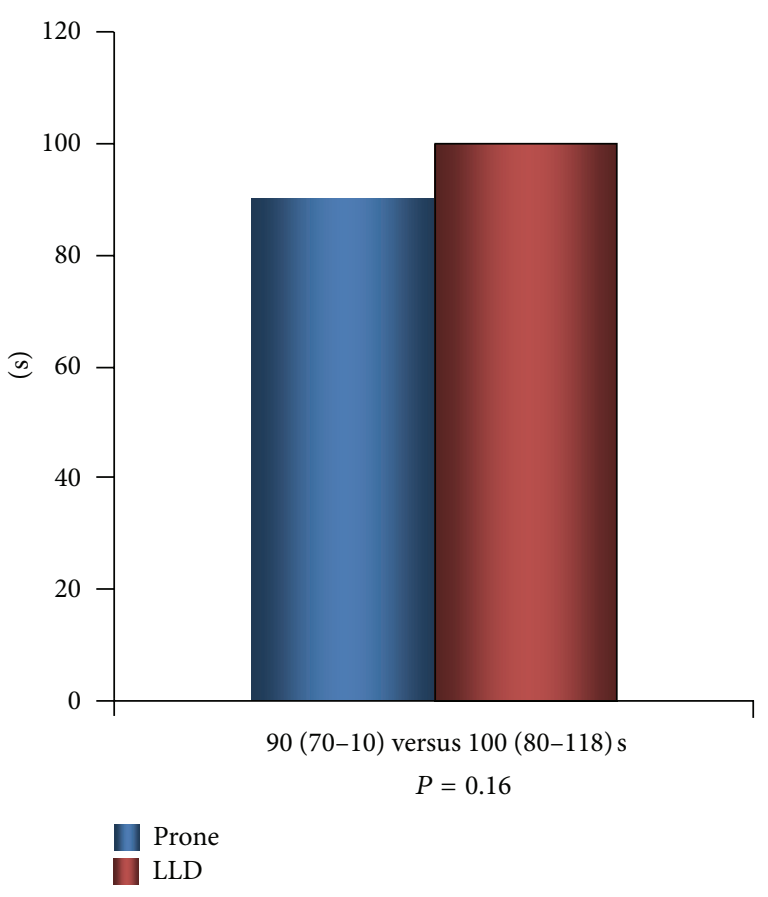

FIGURE 2: Median time from scope insertion to initial ampullary localization in Prone and LLD position. Median (IRQ) time from scope insertion to initial ampullary localization was not significantly different between the prone position and LLD.

localized at 2 minutes, and the endoscope position was stable, but bile duct cannulation was unsuccessful. Attempts to cannulate the bile duct in both short and long positions were not effective due to tangential approach of the sphincterotome to the ampulla. The patient then was repositioned in the prone position which was tolerated after sedation, and in this position the sphincterotome approach to the ampulla improved allowing deep biliary cannulation. In summary, initiation of ERCP in the LLD position for patient comfort does not eliminate the possibility of repeating the procedure in prone position after failed cannulation, provided the patient can tolerate prone position at least temporarily.

Secondary outcomes included time from esophageal intubation with the endoscope to initial ampullary localization and time from initial biliary impaction to free biliary cannulation. These outcomes also were comparable between groups undergoing ERCP in prone versus LLD positions. The median time from esophageal intubation to initial ampullary localization was 110 (80-118) seconds in LLD position and 90 (70-110) seconds in prone position; this difference did not achieve statistical significance $(P=0.16)$ (Figure 2$)$. The median time from ampullary impaction to deep ampullary cannulation was 165 (55-418) seconds in LLD position, and this was not significantly different from the corresponding median time in prone position which was 140 (45-350) seconds $(P=0.54)$ (Figure 3$)$.

Complications occurred in a total of $7 / 59$ patients (11.9\%), with $4 / 39(10.3 \%)$ in the prone position and $3 / 20(15 \%)$ in the LLD position. There was no significant difference in the rate 
TABLE 1

\begin{tabular}{lccc}
\hline & $\begin{array}{c}\text { Prone } \\
(n=39)\end{array}$ & $\begin{array}{c}\text { LLD } \\
(n=20)\end{array}$ & $P$ value \\
\hline ERCP indication & & & \\
$\quad$ Choledocholithiasis; $n(\%)$ & $16(41 \%)$ & $11(55 \%)$ & \\
$\quad$ Malignant stricture; $n(\%)$ & $8(21 \%)$ & $5(25 \%)$ & 0.78 \\
$\quad$ Benign stricture; $n(\%)$ & $3(8 \%)$ & $1(5 \%)$ & \\
$\quad$ Other; $n$ (\%) & $12(31 \%)$ & $3(15 \%)$ & \\
ASA class & & & \\
(1) Healthy & $0(0 \%)$ & $0(0 \%)$ & \\
(2) Mild disease & $26(67 \%)$ & $12(60 \%)$ & \\
(3) Severe disease & $11(28 \%)$ & $4(20 \%)$ & 0.26 \\
(4) Severe threat to life & $2(5 \%)$ & $4(20 \%)$ & \\
(5) Moribund & $0(0 \%)$ & $0(0 \%)$ & \\
Schutz score & & & \\
(1) Simple diagnostic ERCP & $1(3 \%)$ & $0(0 \%)$ & \\
(2) Simple therapeutic ERCP & $9(23 \%)$ & $5(25 \%)$ & \\
(3) Complex diagnostic ERCP & $1(3 \%)$ & $2(10 \%)$ & 0.22 \\
(4) Complex therapeutic ERCP & $19(49 \%)$ & $5(25 \%)$ & \\
(5) Very advanced ERCP & $9(23 \%)$ & $8(40 \%)$ & \\
\hline
\end{tabular}

TABLE 2

\begin{tabular}{lccc}
\hline & $\begin{array}{c}\text { Prone } \\
(n=39)\end{array}$ & $\begin{array}{c}\text { LLD } \\
(n=20)\end{array}$ & $P$ \\
\hline Periprocedural complications; $n(\%)$ & $0(0 \%)$ & $0(0 \%)$ & 1.00 \\
Postprocedural complications; $n(\%)$ & $4(10 \%)$ & $3(15 \%)$ & 0.68 \\
\hline
\end{tabular}

of postprocedural complications in LLD versus prone group $(P=0.68)$. During the procedures, there were no adverse cardiac or respiratory events in any patient. Postprocedural complications were documented in 7 patients: 3 with abdominal pain requiring admission and observation (none had perforation), 1 with bacteremia, 2 with significant bleeding requiring hospitalization, and 1 with pancreatitis (Table 2).

High-quality images were obtained effectively in all patients. As described in Section 2, the initial fluoroscopic positioning was altered for patients in the LLD position, with the fluoroscopic tower rotated media-laterally to achieve anterior-posterior images. However, the initial altered position of the fluoroscopy tower in the LLD group did not limit the effective use of fluoroscopy in any patient, and clear images were achieved with patients both in prone and LLD positions.

\section{Discussion}

ERCP may be poorly tolerated in the customary prone position for a variety of reasons including tense ascites, indwelling percutaneous catheters, advanced pregnancy, abdominal tenderness, or cervical spine disease with limitation of neck rotation. For those patients intolerant of prone positioning, ERCP can be performed in supine or LLD position. ERCP in supine position has been found to be technically feasible, but

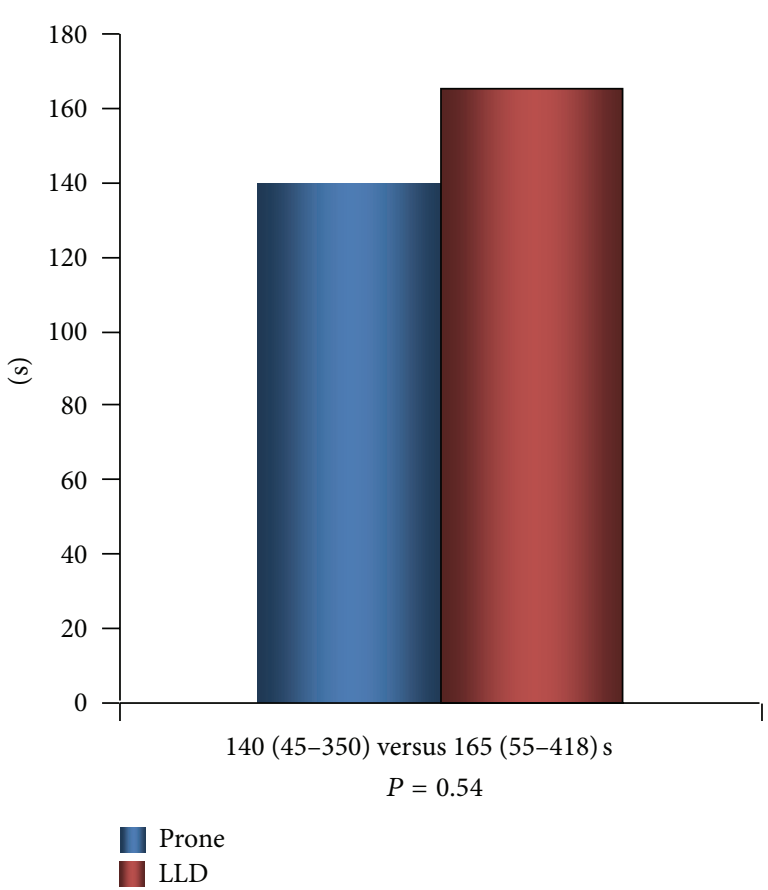

FIgURE 3: Median time from initial ampullary impaction to deep ampullary cannulation in prone and LLD position. Median (IRQ) time from initial ampullary impaction to deep ampullary cannulation was also not significantly different between the prone position and LLD.

the supine position can be uncomfortable for the endoscopist and may result in greater technical difficulty in cannulation or increased rate of complications 4, 5. In theory, ERCP in the LLD position may have advantages over supine positioning. The LLD position is a less radical change from prone position for the endoscopist, resulting in a lesser need for the adjustment of endoscopic technique. LLD position also may allow effective control of secretions by allowing drainage from the oropharynx with gravity rather than requiring frequent suctioning. This is the first study to evaluate the efficacy and safety of ERCP in the left lateral decubitus position as compared to ERCP in prone position.

In this retrospective review, the primary outcome was the biliary cannulation rate of ERCP in the left lateral decubitus position versus the prone position. Cannulation rates proved to be similar in the two groups: there was no statistical difference in free biliary cannulation rates for patients undergoing ERCP in LLD position versus prone position. Two patients who began ERCP in the LLD position did fail initial cannulation, where none failed cannulation in prone position, but both of these patients ultimately did have free biliary cannulation after moving the patient to prone position secondarily. In short, ERCP in the left lateral decubitus position was found to be an effective technical alternative to ERCP in routine prone position for biliary cannulation.

For secondary outcomes, this study compared the time required to perform biliary cannulation and the safety of the procedure in LLD versus the prone position. Time required 
for biliary cannulation was divided into two component time intervals: the time elapsed from esophageal intubation to initial ampullary localization and the time required from ampullary impaction to deep ampullary cannulation. There was no statistically significant difference between LLD and prone positions in the time from esophageal intubation to initial ampullary localization or the time from ampullary localization to deep ampullary cannulation. Numerical trends did favor the prone position with slightly shorter times for ampullary localization and biliary cannulation, which suggests that a larger study might show a statistically significant difference. Specifically, ampullary localization in prone position required a mean of 10 seconds less than in LLD position, and biliary cannulation in prone position required a mean of 25 seconds less than that in the LLD position. However, these differences in time are relatively small compared to the overall time required for ERCP, so it is not clear that any additional time required for ERCP in the LLD position would be clinically meaningful, even if the times were proven to be statistically different by a larger study.

Among endoscopic procedures, ERCP consistently has the highest rate of complications, so any increase in the rate of complications due to altered technique could be clinically significant. However, the results of this study do not suggest that ERCP in LLD position increases the risk of complications as compared to ERCP in prone position. Specifically, there were no periprocedural cardiopulmonary or sedation-related complications in either group. Seven patients did experience postprocedural complications, but there was no significant difference between the LLD and prone groups. All patients who experienced postprocedural complications were treated successfully and discharged in a stable condition.

Interestingly, while this study demonstrates that ERCP can be performed effectively and safely in the LLD position, thereby offering a comfortable procedure for the patient with abdominal or cervical disorders, the endoscopist may find it technically challenging to perform ERCP in the LLD position. For the two patients in this study who had failed cannulation in the LLD position, it proved difficult to center and stabilize the ampulla in the prone position for effective cannulation, and free biliary cannulation was achieved only in prone position. The body position of the endoscopist also is less favorable in many of the ERCP cases done in LLD position: the endoscopist frequently must turn clockwise as much as 90 degrees away from the patient to center the ampulla and allow stable positioning of the endoscope for cannulation. This bodily rotation of the endoscopist away from the patient impairs the endoscopist's view of the endoscopy monitor and may require the use of a second monitor situated behind the endoscopist, which may not be available in every endoscopic unit.

The primary limitations of this study are its retrospective design and relatively small sample size. A prospective, randomized controlled trial would be helpful to definitively compare the cannulation rate, time required for ERCP, and complications in LLD versus prone position. However, because patients with abdominal or cervical disorders are often a relatively small subset of all patients undergoing ERCP, it would require a large volume of ERCP studies to achieve adequate power to differentiate outcomes among this subset of patients. A larger study also might be useful to determine if the trends noted in this study towards comparatively decreased biliary cannulation rates and longer procedural times for ERCP in LLD position would be confirmed as significant differences from patients undergoing ERCP in prone position. Another potential limitation to the generalization of these findings might be the inclusion of procedures completed by single senior endoscopist. However, the inclusion of cases from a single endoscopist experienced in advanced therapeutic endoscopy did reduce the variance due to endoscopist experience and allowed a more direct comparison of the clinical utility of the prone and LLD positions. Additionally, because these studies were often completed in critically ill patients (33\% of prone and $40 \%$ of LLD patients were ASA 3 or above) and the procedures were technically difficult (72\% of prone and $65 \%$ of LLD patients were rated as Schutz score 4 or above for difficulty of ERCP), they are most likely to be performed by more experienced endoscopists. However, the applicability of our findings to gastroenterologists performing ERCP in the community setting is unclear.

In conclusion, ERCP can be effectively and safely performed in the left lateral decubitus position with no significant diminution in biliary cannulation rate, prolongation of the procedure, or increase in complications. However, there was a trend towards greater success in cannulation and shorter procedural time in the prone position compared to the LLD position. ERCP in the left lateral decubitus position therefore should not be considered a replacement for customary prone positioning, but may be a practical alternative for cases in which the endoscopist determines that the standard prone position would be problematic for the patient or technically challenging.

\section{Conflict of Interests}

The authors have no conflict of interests to declare.

\section{References}

[1] A. Tringali, M. Mutignani, A. Milano, V. Perri, and G. Costamagna, "No difference between supine and prone position for ERCP in conscious sedated patients: a prospective randomized study," Endoscopy, vol. 40, no. 2, pp. 93-97, 2008.

[2] A. Das, "Performing an ERCP with the patient in the supine position: necessity is the mother of improvisation," Gastrointestinal Endoscopy, vol. 67, no. 7, pp. 1044-1045, 2008.

[3] F. Froehlich, "Patient position during ERCP: prone versus supine. What about left lateral throughout?" Endoscopy, vol. 38, no. 7, art, 2006.

[4] L. E. V. V. C. Ferreira and T. H. Baron, "Comparison of safety and efficacy of ERCP performed with the patient in supine and prone positions," Gastrointestinal Endoscopy, vol. 67, no. 7, pp. 1037-1043, 2008.

[5] V. Terruzzi, F. Radaelli, G. Meucci, and G. Minoli, "Is the supine position as safe and effective as the prone position for 
endoscopic retrograde cholangiopancreatography? A prospective randomized study," Endoscopy, vol. 37, no. 12, pp. 1211-1214, 2005.

[6] S. M. Schutz and R. M. Abbott, "Grading ERCPs by degree of difficulty: a new concept to produce more meaningful outcome data," Gastrointestinal Endoscopy, vol. 51, no. 5, pp. 535-539, 2000.

[7] T. M. Berzin, S. Sanaka, S. R. Barnett et al., "A prospective assessment of sedation-related adverse events and patient and endoscopist satisfaction in ERCP with anesthesiologistadministered sedation," Gastrointestinal Endoscopy, vol. 73, no. 4, pp. 710-717, 2011. 


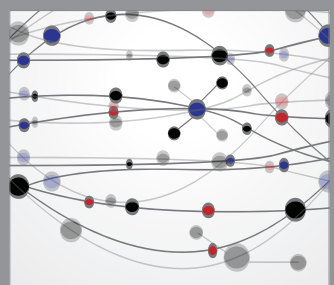

The Scientific World Journal
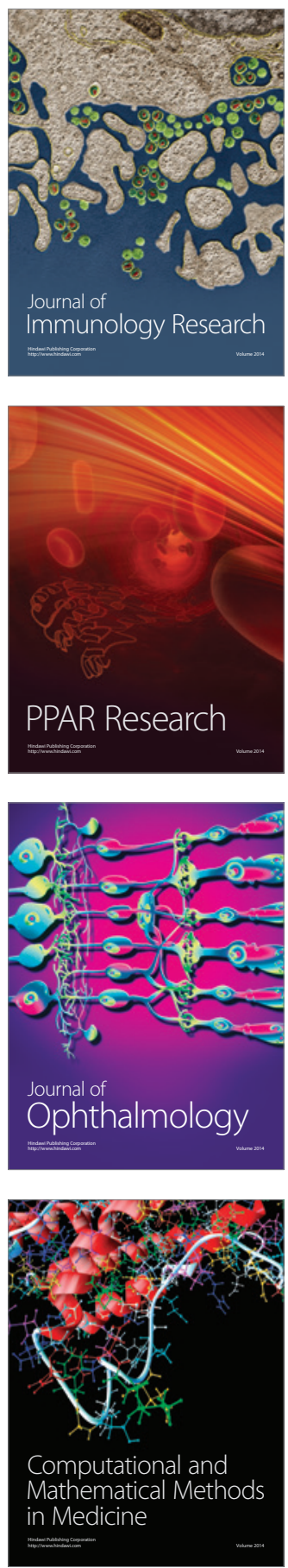

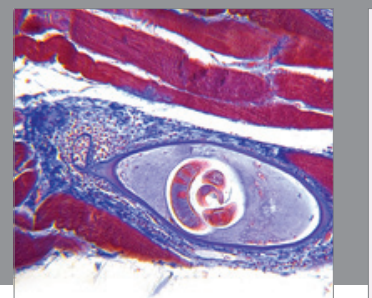

Gastroenterology

Research and Practice
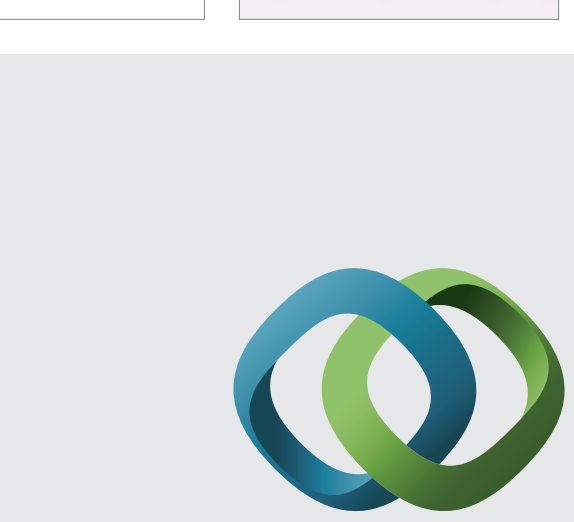

\section{Hindawi}

Submit your manuscripts at

http://www.hindawi.com
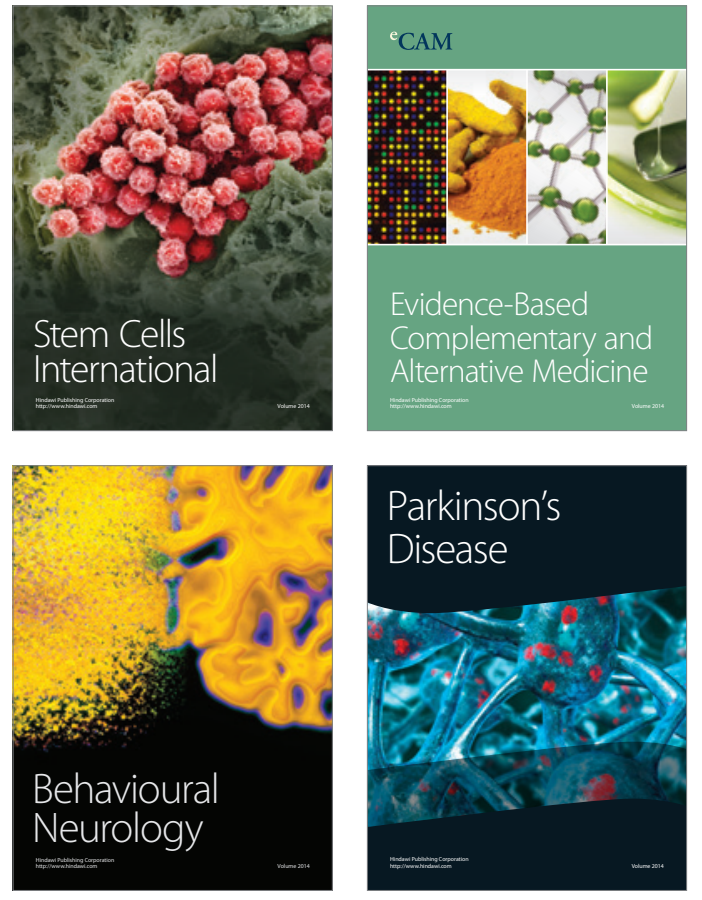
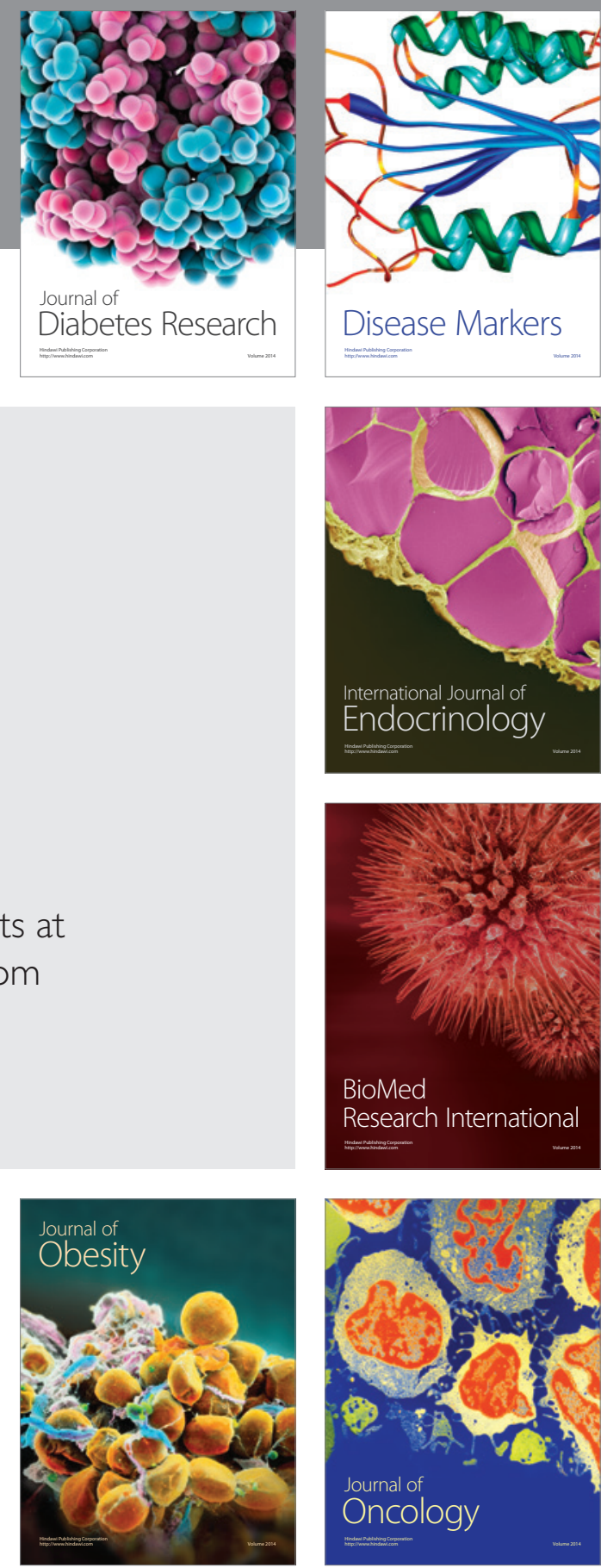

Disease Markers
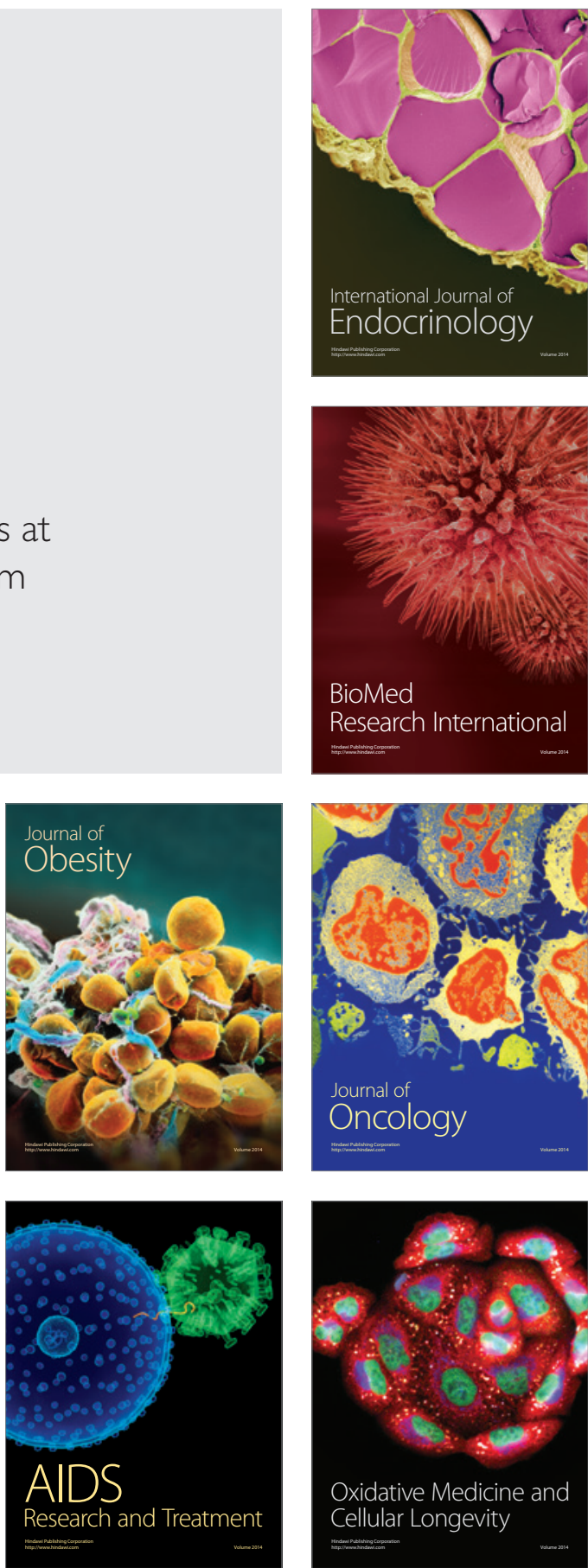